\title{
Mettre des limites à notre pulsion prométhéenne? Possible?
}

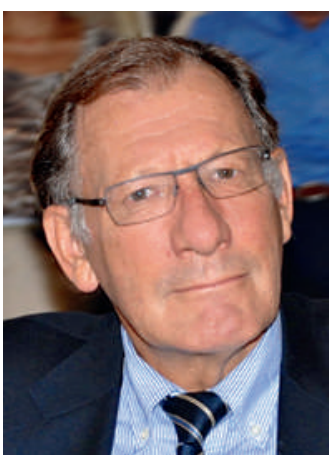

Jean Martin
Je suis de ceux qui croient qu'il n'est pas souhaitable de faire tout ce que la science et les techniques permettent d'envisager, qu'il faut élaborer et faire observer des limites, des bornes. Par exemple, même si certains futuristes l'annoncent pour bientôt, je crois qu'il faut refuser le clonage humain. Cela étant, le caractère prométhéen de l'Homme ne peut être nié. Certes, dans les forêts vierges d'Amérique équatoriale, d'Afrique ou d'ailleurs, il y a eu et il y a par endroits des sociétés vivant dans une entente respectueuse avec la nature, en harmonie avec elle, sans semble-t-il que la volonté de dominer et d'étendre son emprise sur le milieu soit un but collectif important.

Ce sujet m'a occupé l'esprit durant 2013 lors de deux voyages: l'un en Grèce, visitant des sites historiques, à Mycènes entre autres: ouvrages cyclopéens datant de 1200 à 1500 ans avant J.-C.; l'autre au Brésil, occasion de mieux toucher du doigt les péripéties de l'évolution de cet immense pays au cours des derniers siècles. Comment ne pas rester frappé, comment ne pas (dans une certaine mesure au moins) admirer la volonté de l'Homme d'accroître son expérience, d'inventer, de construire, de découvrir et surmonter les obstacles (y compris c'est vrai en faisant la guerre à des congénères).

En bioéthique, la vitesse de l'évolution des moyens techniques de procréation médicalement assistée (PMA) est impressionnant (on se souvient que le premier bébé-éprouvette, Louise Brown, est né en 1978). Et je reste songeur en voyant avec quelle rapidité nous nous habituons, moi et les autres, à des modalités qui il y a trente ans paraissaient pour le moins discutables voire à proscrire. Je rappelle que, avant que soit confiée à la Confédération la compétence de légiférer en cette matière, deux cantons avaient passé des lois prohibant toute pratique de la PMA; aujourd'hui une tentative dans ce sens connaîtrait un échec cuisant. Et les méthodes de PMA sont de plus en plus étonnantes voire acrobatiques, et de plus en plus routinisées.

Ces poussées prométhéennes sont-elles une fatalité? Allons-nous toujours à l'avenir faire tout ce que la bioscience permet? Je voudrais des limites mais ne suis pas sûr qu'on puisse arrêter le train sur les rails. Quelle est alors la place de l'éthique? Des instances comme la Commission nationale d'éthique et ses homologues ailleurs ont pour tâche de dire, sur la base d'évaluations philosophiques, juridiques, sociales et scientifiques, où à leur avis il convient de mettre des barrières et pourquoi. Ces commissions toutefois ont un rôle consultatif seulement et non de décision - et c'est bien ainsi.

Les décisions elles sont le fait du politique selon les modalités de chaque pays (démocratie directe chez nous, avec ses grandeurs et ses servitudes). Le politique va apprécier puis décider si on peut fixer dans la loi les règles proposées par l'éthique (cas échéant dans quelle mesure) et dans quelles conditions. Ceci notamment au vu du régime juridicopolitique en vigueur, en particulier: les droits fondamentaux reconnus à chacun, le monopole de la contrainte légitime accordé à l'Etat, le périmètre dudit monopole, le fait que ce n'est pas le rôle de l'Etat de «faire la morale». De là découle qu'il y aura toujours un hiatus entre ce qui peut être éthiquement recommandé et ce qui est décidé au plan sociétal.

Des éthiciens engagés, on peut les comprendre, s'étonnent voire sont choqués de ce que leurs conseils ne sont pas toujours suivis. Dits conseils peuvent être excellents mais il reste que ce n'est pas à eux d'en décider. De plus, si c'était le cas, le risque serait d'aller vers une éthicocratie, pas très éloignée d'une théocratie - et on voit bien, dans l'Histoire passée comme actuelle, à quelles dérives mènent ces régimes (étant entendu que les systèmes marxistes étaient du même registre).

Je cite souvent la formule «La liberté ne vaut que par les limites qu'on lui met». Etre civilisé c'est mettre des limites: au pouvoir de l'Etat sur les personnes, aux contraintes indues d'une personne sur une autre. Allons-nous perdre notre caractère civilisé si nous refusons d'envisager des interdictions quant à l'application des avancées techniques, dans le domaine de la PMA par exemple? Dans certains cas, oui je le crains; dans d'autres pas.

La vie en société est fondée sur un corpus juridique (plus ou moins formalisé selon les cultures d'ailleurs). Les lois sont votées par le parlement et elles peuvent en Suisse être invalidées par le peuple. L'expérience montre qu'une majorité populaire peut voter des lois contraires au droit supérieur internationalement reconnu. C'est l'enjeu. Peut-on compter sur la sagesse des parlementaires, respectivement des citoyens, pour poser au bon endroit des limites aux applications discutables de la bioscience? Je ne sais pas.

Jean Martin, membre de la Commission nationale d'éthique (il s'exprime ici à titre personnel) et de la rédaction 